\title{
Pedaleando hacia el futuro: Una oportunidad para incrementar y mantener la asistencia escolar de niñas adolescentes encomunidades indígeneas del árearural en Guatemala
}

Paola Broll

Population Council

Follow this and additional works at: https://knowledgecommons.popcouncil.org/departments_sbsr-pgy

Part of the Demography, Population, and Ecology Commons, Family, Life Course, and Society Commons, Gender and Sexuality Commons, and the International Public Health Commons How does access to this work benefit you? Let us know!

\section{Recommended Citation}

Broll, Paola. 2018. "Pedaleando hacia el futuro: Una oportunidad para incrementar y mantener la asistencia escolar de niñas adolescentes encomunidades indígeneas del árearural en Guatemala," brief. Guatemala City: Population Council. 


\section{PEDALEANDO HACIA EL FUTURO:}

UNA OPORTUNIDAD PARA INCREMENTAR Y MANTENER LA ASISTENCIA ESCOLAR DE NIÑAS ADOLESCENTES ENCOMUNIDADES INDÍGENEAS DEL ÁREARURAL EN GUATEMALA

PAOLA BROLL

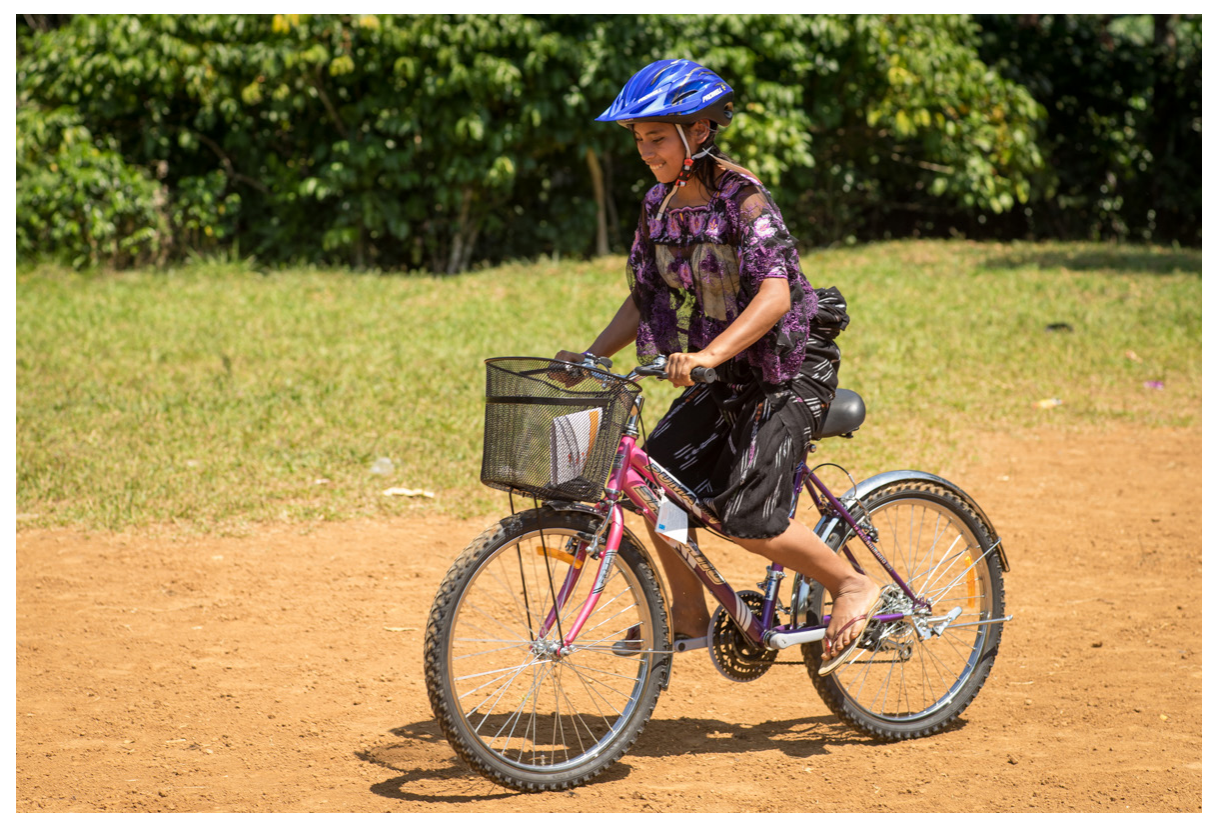

Participante de Pecajbá, Chisec, Alta Verapaz camino al instituto de estudios secundarios a 7 km de distancia de su casa.

Population Council Guatemala, con el apoyo de UNFPA y otros donantes, desarrolló la estrategia de expansión y escala de Abriendo Oportunidades $(\mathrm{AO})$ a través de conglomerados geográficos con abordaje ecológico. AO fue implementado en cuatro municipios: Chisec (Alta Verapaz), San Luis, Dolores y Poptún (Petén), alcanzando a 4,425 participantes. La provisión de 250 bicicletas es un componente adicional de AO para probar su efectividad en el aumento de movilidad y la asistencia escolar durante noviembre 2016 a octubre 2017 en los cuatro municipios. Las bicicletas fueron entregadas en 30 comunidades que no tienen escuelas de estudios secundarios.

El Population Council es una organización no gubernamental internacional sin fines de lucro que realiza investigaciones y ofrece soluciones para mejorar la vida de todo el mundo. La investigación realizada por Population Council en Guatemala demostró que los programas sociales fallan sistemáticamente en llegar a la población más vulnerable pues no están diseñados específicamente para atender sus necesidades. ${ }^{1}$

\section{RESULTADOS PRINCIPALES}

1. La dotación de 250 bicicletas a adolescentes que participan en el Programa Abriendo Oportunidades incrementó en $24 \%$ la reinserción escolar del ciclo 2016 al ciclo 2017.

2. El $97 \%$ de las participantes completó el ciclo escolar 2017.

3. La intención de estudio de las 250 participantes aumentó del $76 \%$ (ciclo escolar 2017) al 97\% (ciclo escolar 2018).

4. Las adolescentes desean seguir estudiando cuando tienen un medio de transporte que facilita su movilidad al centro de estudios más cercano.

5. El $51 \%$ de las participantes aumentó su movilidad con la bicicleta.

6. El aumento de movilidad con las bicicletas, permitió que las participantes continuaran sus estudios secundarios, ocuparan espacios públicos de intercambio comercial, fortalecieran su capital social y tuvieran acceso a actividades de recreación.

\footnotetext{
1 The Adolescent Experience In-Depth: Using Data to Identify and Reach the Most Vulnerable Young People: Guatemala 2002/2006. New York: Population Council, 2009.

http://www.popcouncil.org/uploads/pdfs/PGY_AdolDataGuides/Guatemala2002-06.pdf.
}

Population Council realiza investigación y propone soluciones para mejorar las vidas al rededor del mundo. Grandes ideas basadas en evidencia: nuestro modelo para el cambio global. popcouncil.org 
En respuesta, Population Council creó el programa Abriendo Oportunidades para alcanzar a las niñas adolescentes indígenas en mayores condiciones de vulnerabilidad en comunidades rurales de Guatemala ${ }^{2}$. Desde 2004, AO ha formado grupos de niñas adolescentes, divididas por edades, que se reúnen una vez por semana en un espacio seguro para desarrollar sesiones de la Guía Curricular de $\mathrm{AO}^{3}$. Las sesiones son facilitadas por mentoras quienes son mujeres de las mismas comunidades entre 18 y 24 años que se convierten en agentes de cambio social. El resultado de este esfuerzo es el incremento en la autoestima de las participantes, el impulso de liderazgo de niñas, adolescentes y mujeres y la promoción de la equidad de género. ${ }^{4}$

AO involucra a los líderes de la comunidad, aumenta las redes de apoyo social de las niñas adolescentes y las conecta con modelos alternos de mentoras con quienes aprenden habilidades prácticas que las prepara en la transición a la vida adulta y a asumir funciones de liderazgo. Para el UNFPA es prioritario llegar a los adolescentes y jóvenes marginados y desfavorecidos, especialmente las niñas, porque millones de ellas viven en la pobreza extrema. La iniciativa de niñas adolescentes (AAG) del UNFPA apoyó para trabajar de manera focalizada e integrada en beneficio de las niñas adolescentes en riesgo de unión infantil y embarazo precoz.

\section{ACTIVIDADES \& RESULTADOS}

En noviembre de 2016, 1,188 participantes de AO realizaron la sesión de Plan de Vida. En esta sesión cada adolescente establece cinco metas en su vida en las áreas personal, familiar, estudio/trabajo, económica y comunitaria. Las metas se definen a corto, mediano y largo plazo y las presenta en una reunión con madres, padres y autoridades locales. La revisión de los Planes de Vida indicó que de las 1,188 participantes, 976 (82\%) sí estudiaron en el año 2016 y 212 (18\%) no lo hicieron (Gráfico 1). Los criterios de selección de participante para recibir una bicicleta fueron los siguientes:

1. Distancia de la casa de la participante al centro de estudio más cercano;

2. Medio de transporte de la casa de la participante al centro de estudio más cercano;

3. Costo del transporte de la casa de la participante al centro de estudio más cercano; y

4. Tiempo invertido en caminar, si es el caso, de la casa de la participante al centro de estudio más cercano.

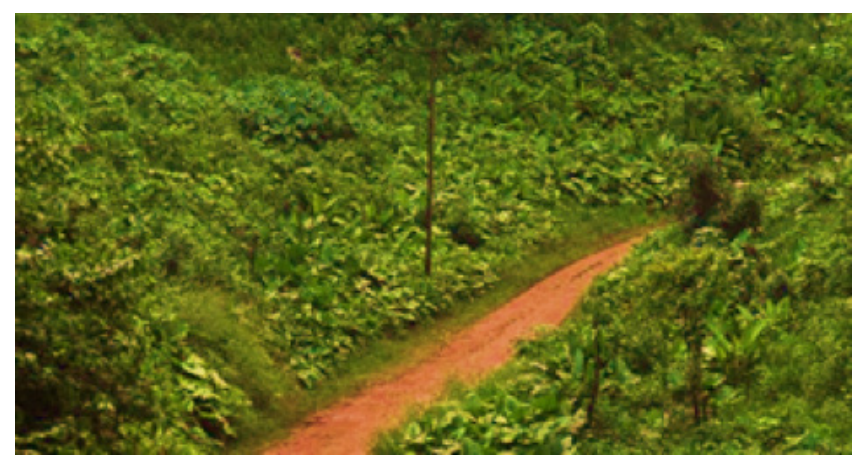

Los caminos del área rural son, en su mayoría, solitarios, de tierra y rodeados de siembras. Las participantes recorren -en promedio- $10 \mathrm{~km}$ diarios para estudiar en un centro comunitario de educación escolar o extra escolar.

GRÁFICO 1. PORCENTAJE DE LAS 1,188 PARTICIPANTES DE AO QUE ESTUDIARON EN EL AÑO 2016

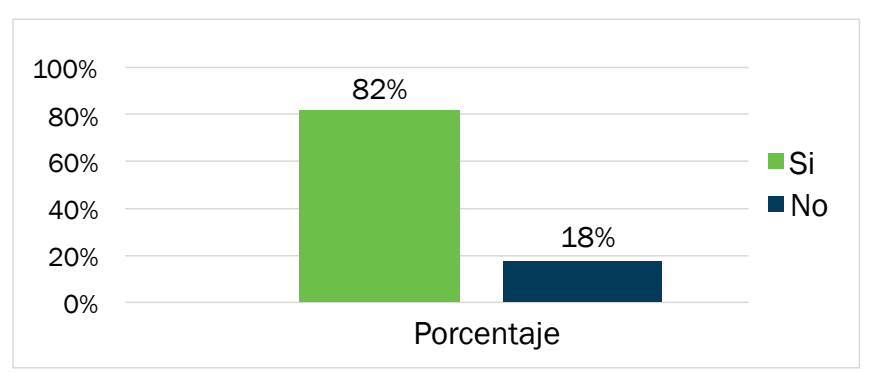

Fuente: Base de datos de monitoreo de AO. Sesión Plan de Vida. Noviembre 2016 GRÁFICO 2. PORCENTAJE DE LAS 250 PARTICIPANTES
SELECCIONADAS PARA RECIBIR BICICLETA CON
INTENCIÓN DE ESTUDIO PARA EL CICLO ESCOLAR 2017

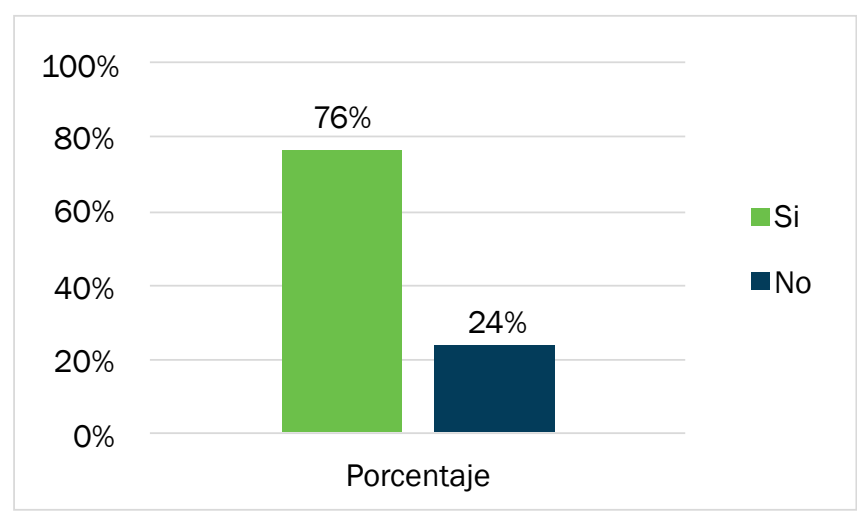

Fuente: Base de datos de monitoreo de AO. Noviembre 2016

\footnotetext{
${ }^{2}$ https://www.girlsnotbrides.org/wp-content/uploads/2016/02/Case-Study-Empower-girls-Population-Council-Guatemala.pdf.

${ }^{3}$ http://www.popcouncil.org/research/abriendo-oportunidades-program-integrated-curriculum-guide.

${ }^{4}$ Cushman, Nicole. 2010. Guatemala rising, one girl at a time. Findings from the 2009-2010 Abriendo Oportunidades evaluation. EEUU: Population Council.
} 
De las 212 participantes que no estudiaron en el año 2016, 59 cumplieron los criterios de selección y dijeron no tener intención de estudio para el 2017 por falta de recursos económicos y distancia a recorrer para llegar al centro de estudios. Durante las visitas de monitoreo de noviembre y diciembre 2016 se exploró la posibilidad, a través de visitas domiciliarias, de la reinserción escolar a estudios secundarios del 24\% (59) de las 250 seleccionadas para recibir bicicleta; pues las otras 191 participantes (76\%) indicaron que sí tenían intención de estudio para el ciclo escolar 2017 (Gráfico 2). La entrega de las 250 bicicletas (228 en Alta Verapaz y 22 en Petén) fue en febrero y marzo de 2017, coincidiendo con la inscripción al ciclo escolar 2017 y logrando la inscripción del $100 \%$ de las participantes del proyecto.

El equipo de Population Council monitoreó actividades mensualmente con el apoyo en campo de cuatro mentoras. En Alta Verapaz se visitaban 30 comunidades con 5 institutos secundarios y 10 centros de estudio del Programa Modalidades Flexibles . En Petén se visitaban 20 comunidades con un centros de estudio del Programa Modalidades Flexibles. Mensualmente se monitoreó el avance en los estudios; Ios retos enfrentados al tener bicicletas; los lugares visitados -dentro y fuera de su comunidad- y el propósito de las visitas realizadas con la bicicleta; las personas que usan la bicicleta; si alguna vez que quiso usar la bicicleta no pudo porque la tenía alguien más; los gastos incurridos en reparaciones de la bicicleta; y las razones para abandonar los estudios.

Los datos de monitoreo muestran que las participantes cambiaron su intención de estudio del 76\% para el ciclo 2017 al 97\% para el ciclo 2018 (Gráfico 3). Con estos datos se infiere que al tener un medio de transporte que les facilite la movilidad para llegar al centro de estudios, las jóvenes desean continuar estudiando.

Al inicio del proyecto el 49\% reportó moverse dentro o fuera de la comunidad. Al finalizar el proyecto, el 100\% reportó algún tipo de movilidad. Información recabada durante los monitoreos indicó que las participantes aumentaron su movilidad en un $11 \%$ dentro y $37 \%$ fuera de sus comunidades (Gráfico 4). El 74\% de las participantes indicó que se movilizó hacia lugares que representan actividades económicas relacionadas con las tareas del hogar, como el molino, mercado y la tienda de víveres. El $66 \%$ coincidió en que la bicicleta las ayudó a poder visitar a sus amigas, fortaleciendo su red de apoyo y capital social en actividades de recreación como juego de pelota o bañarse en el río (Gráfico 5).
GRÁFICO 3. COMPARACIÓN DE PORCENTAJES DE INTENCIÓN DE ESTUDIO DE LAS 250 PARTICIPANTES PARA LOS CICLOS ESCOLARES 2017 Y 2018

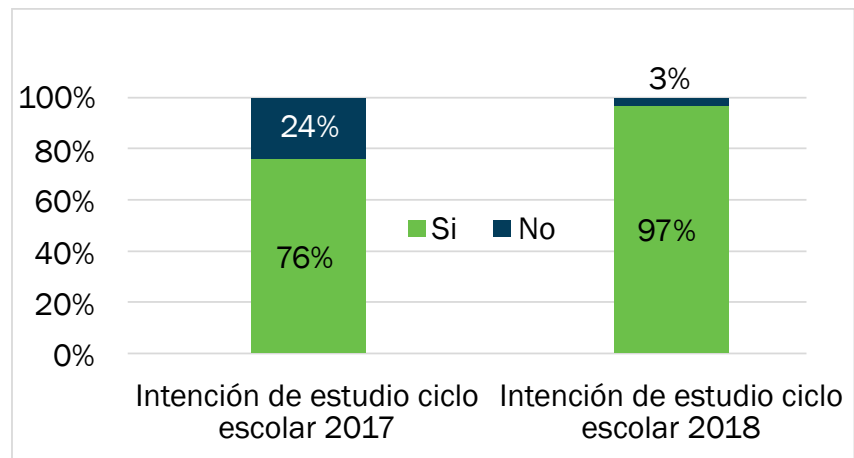

Fuente: Base de datos de monitoreo de AO. Octubre 2017.

GRÁFICO 4. COMPARACIÓN DE LOS PORCENTAJES DE MOVILIDAD DENTRO Y FUERA DE LA COMUNIDAD, AL INICIO Y AL FINAL DEL PROYECTO

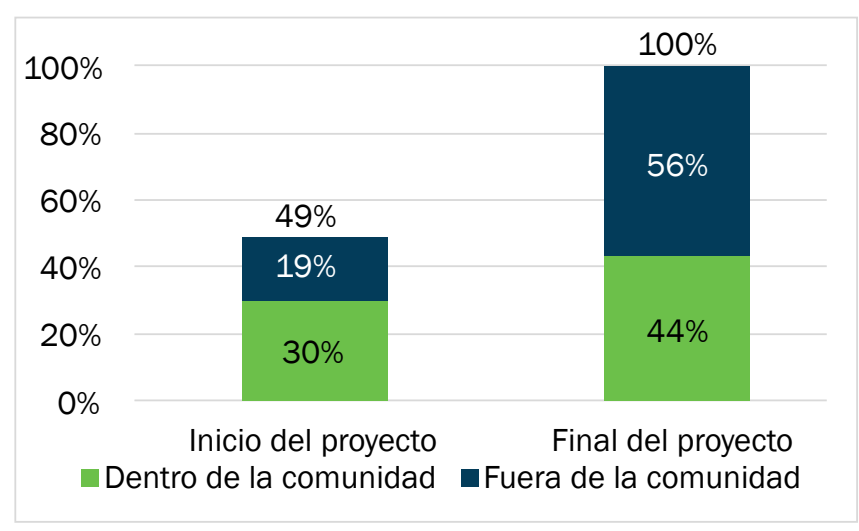

Fuente: Base de datos de monitoreo de AO. Octubre 2017.

GRÁFICO 5. COMPARACIÓN DE PORCENTAJES DE LOS LUGARES MÁS VISITADOS EN BICICLETA POR LAS 250 PARTICIPANTES

\section{0\%: Centro de estudios}

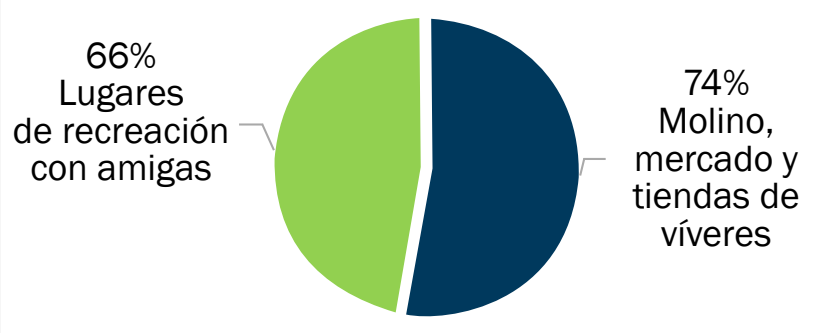

Fuente: Base de datos de monitoreo de AO. Octubre 2017.

${ }^{5}$ http://estadistica.mineduc.gob.gt/SNIE/eficiencia.html 
La mayoría relató que tuvieron que esconderla de la entrada del centro de estudios para que los compañeros hombres no se las quitaran. Muchas tuvieron que prestársela a sus padres y hermanos y tenerla disponible para llegar al centro de estudios.

\section{CONCLUSIONES Y RECOMENDACIÓN}

1. Las largas y peligrosas distancias que las adolescentes recorren a pie para llegar a un centro de estudios secundario, favorecen las ausencias escolares y el abandono de los estudios.

2. El proyecto fue exitoso en el aumento de la movilidad, reinserción y finalización del ciclo escolar 2017 de sus participantes.

3. El proyecto demostró éxito al aumentar la intención de estudio del 76\% (ciclo escolar 2017) al 97\% (ciclo escolar 2018).

4. En las comunidades en donde se entregaron bicicletas, nunca una mujer había tenido un medio de transporte propio. La recepción de la bicicleta les abrió oportunidades a las participantes del proyecto para poder ir al centro de estudios más cercano, visitar comunidades vecinas para actividades económicas y gozar de actividades de recreación con amigas.

5. En Chisec, Alta Verapaz, la tasa de conservación nacional del sistema escolarizado para el 2016 fue del 79.8\%5. El proyecto logró una tasa de conservación del 98\%, pues de las 228 participantes solo 4 abandonaron los estudios.

6. El proyecto logró una tasa de conservación del $75 \%$ notando que las participantes estudiaron en el sistema extraescolar que no cuenta con datos de conservación nacional.



Una joven adolescente recibe una de las 250 bicicletas entregadas a través del Programa Abriendo Oportunidades ${ }^{\circledast}$. 